\title{
Kuiper Belt Dust Grains as a Source of Interplanetary Dust Particles
}

\author{
Jer-Chyl Liou and Herbert A. Zook \\ SN3, NASA Johnson Space Center, Houston. Texas 77058 \\ E-mail: liou@sn3.jsc.nasa.gov. \\ AND \\ Stanley F. Dermott \\ Department of Astronomy, University of Florida, Gainesville. Florida 32511 \\ Received August 25. 1995: revised May 16, 1996
}

The recent discovery of the so-called Kuiper belt objects has irompted the idea that these objects produce dust grains that nat contribute significantly to the interplanetary dust populathon. In this paper. the orbital evolution of dust grains, of diameters 1 to $9 \mu \mathrm{m}$, that originate in the region of the Kuiper belt is studied by means of direct numerical integration. Gravitational forces of the Sun and planets, solar radiation pressure, as well as Poynting-Robertson drag and solar wind drag are included. The interactions between charged dust grains and solar magnetic field are not considered in the model. Because of the effects of drag forces, small dust grains will spiral toward the Sun once they are released from their large parent bodies. This motion leads dust grains to pass by planets as well as encounter numerous mean motion resonances associated with planets. Our results show that about $80 \%$ of the Kuiper belt grains are ejected from the Solar System by the giant planets, while the remaining $20 \%$ of the grains evolve all the way to the Sun. Surprisingly, the latter dust grains have small orbital eccentricities and inclinations when they cross the orbit of the Earth. This makes them behave more like asteroidal than cometary-type dust particles. This also enhances their chances of being captured by the Earth and makes them a possible source of the collected interplanetary dust particles; in particular, they represent a possible source that brings primitive/organic materials from the outer Solar System to the Earth.

When collisions with interstellar dust grains are considered, however, Kuiper belt dust grains around $9 \mu \mathrm{m}$ appear likely to be collisionally shattered before they can evolve toward the inner part of the Solar System. The collision destruction can be applied to Kuiper belt grains up to about $50 \mu \mathrm{m}$. Therefore, Kuiper belt dust grains within this range may not be a significant part of the interplanetary dust complex in the inner Solar Sistem. 1996 Academic Press, Inc.

\section{INTRODUCTION}

Asteroids and comets have long been considered the wo major sources that contribute dust particles to the
Solar System interplanetary space (e.g.. Leinert and Grün 1990. Levasseur-Regourd e: al. 1991). However. since the jiscovery of the first iran-neptunian obiect Jewilt and Luu 1992. 1993) and the sull ongoing discoveries of such objects (see Jewitt and Luu 1995 for an up-to-date summary), it has been proposed that small dust particles produced from these so-called “Kuiper belt objects" may contribute significantly to the interplanetary dust population that constitutes the zodiacal cloud (e.g., Flynn 1994). They may also be an important source of the interplanetary dust particles (IDPs) collected in the stratosphere by highaltitude airplanes. The fact that the meteoroid detectors on board Pioneer 10 and 11 recorded impacts at a nearly constant rate up to $18 \mathrm{AU}$ (no data available beyond that) from the Sun (Humes 1980) also suggests a possible dust grain source in the outer Solar System.

Three key questions must be answered before we can estimate the contribution of Kuiper belt (KB) dust grains to the interplanetary meteoritic complex: (1) What is the dust production rate in the region of the KB? (2) Can those grains evolve all the way to the inner part of the Solar System? (3) What is the collisional lifetime of those dust grains when interstellar dust particles are considered? Question 1 requires the knowledge of, in addition to the total population of objects in the KB. for example, the size distribution, composition. and strength of the KB objects. Based on the study by Jewitt and Luu (1995), there are $\sim 35,000$ objects with diameters larger than $100 \mathrm{~km}$ between 30 and 50 AU. Recent observations from the Hubble Space Telescope (HST) also suggest that there are more than $2 \times 10^{8}$ Halley-sized objects in the KB (Cochran et al. 1995). This population is, in principle. capable of producing a large amount of dust (for comparison, there are only about 250 asteroids with diameters larger than $100 \mathrm{~km}$ in the main asteroid belt). A recent collision model of the $\mathrm{KB}$ objects also predicts a total dust production rate 
from $9 \times 10^{8}$ to $3 \times 10^{11} \mathrm{~g} \mathrm{sec}^{\prime}$ (Stern 1995. 1996). This dust cloud may even be visible in the infrared wavelength (Backman et al. 1995: Stern 1996). Comparing the dust production rate in the $\mathrm{KB}$ with the $10^{-} \mathrm{g} \mathrm{sec}^{-1}$ dust needed to maintain the zodiacal cloud in a quasi-equilibrium state (Whipple 1967), it is obvious that the importance of the $\mathrm{KB}$ dust grains in the interplanetary dust complex cannot be overlooked. Based on that, our objectives in this paper are to answer the second and third questions with direct numerical integrations of the orbital evolution of dust grains and apply a simple collision model based on the best available interstellar dust grain spatial density (flux) data. In our numerical calculations, we simulate the actual orbital evolution of the dust grains by including the gravitational perturbations due to the Sun. seven planets (Mercury and Pluto are not included). radiation pressure. Poynting-Robertson (PR) drag. and solar wind drag. We do these calculations for dust grains ranging from 1 to $9 \mu \mathrm{m}$ in diameter. Our collisional model is based in the recent measurement of the mass and flux of

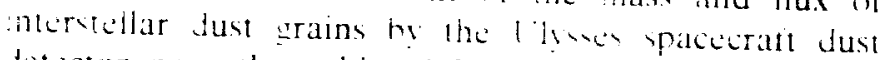
detector near the orbit of Jupiter rirun it al. 1993). We assume that this interstellar dust spatial density is the same throughout the Solar System and remains constant in time. We neglect the effect of the fluctuating solar wind magnetic field force on these interstellar particles even though, for the smaller grains. it may often exceed the solar gravitational force.

Because of PR and solar wind drag. small dust grains will spiral toward the Sun once they are released from their large parent bodies (e.g.. Wyatt and Whipple 1950. Burns et al . 1979). This orbital evolution leads dust grains to pass by planets as well as encounter numerous mean motion resonances (MMRs) associated with planets. Based on our results, trapping into exterior MMRs with Neptune and. to a lesser extent, Uranus dominates the orbital evolution of those dust grains. Secular resonances are related to the precessions of orbits of the dust and planets involved (e.g.. Knežević et al. 1991). When a small dust grain is spiraling toward the Sun. the time it takes to pass by a secular resonance location is short compared with the orbital precession time scale of planets. Unless at dust grain is trapped in an MMR, the effect of secular resonances is not important. Gravitational scattering by Saturn or Jupiter usually controls their final fate. Approximately $80 \%$ of the dust grains in our numerical calculations eventually were scattered out of the Solar System before reaching the inner part of the Solar System. The remaining $20 \%$ of the dust grains were able to pass by Saturn and Jupiter and completed their journeys all the way to the Sun.

When collisions with interstellar dust grains are considered. however, KB dust grains around $9 \mu \mathrm{m}$ appear likely to be collisionally shattered before they can evolve toward the inner part of the Solar System. Thus. collisions may limit the role of KB dust grains in contributing to the zodiacal light.

Our numerical calculations are described in Section 2. The orbital evolution of several typical dust grains is presented in Section 3. A statistical description of results is in Section 4. Our collisional model is in Section 5. The significance of KB dust grains in the inner Solar System is discussed in Section 6.

\section{NUMERICAL CALCULATIONS}

The orbital evolution of dust grains in the Solar System is affected by the radial solar radiation pressure force, by gravitational forces due to the Sun and planets, and by PR and solar wind drag forces. The equation of motion of a dust grain with geometric cross section $A$ and mass $m$ moving under the influence of the gravitational forces of the Sun, seven planets. the radiation pressure force, and the urag forces can be written as (e.g.. Burns el al. 1979 (justafson 1994 )

$$
\begin{aligned}
m \dot{\mathbf{v}}= & -\left[\frac{G \cdot M \cdot m}{r_{h}^{3}}\right] \mathbf{r}-\sum_{n=1}^{\infty} \frac{G M_{n} m}{r_{n}^{3}} \mathbf{r}_{n} \\
& +(S A / c) Q_{\mathrm{pr}}\left[\left(1-(1+s w) \frac{\mathbf{v} \cdot \mathbf{r}_{\mathrm{s}}}{c r_{\mathrm{s}}}\right) \hat{\mathbf{r}}_{\mathrm{s}}-(1+s w) \frac{\mathbf{v}}{c}\right]
\end{aligned}
$$

where $\mathbf{v}$ is the velocity vector of the particle, $\hat{\mathbf{r}}_{\mathrm{b}}$ is its unit position vector with respect to the Sun, and $M_{0}$ is the Sun's mass, while $M_{\mathrm{n}}$ and $\mathbf{r}_{\mathrm{n}}$ are the mass of the $n^{\text {th }}$ planet and the position vector of the particle with respect to that planet. $S, c$, and $Q_{p r}$ are the solar energy flux density, speed of light, and radiation pressure coefficient. respectively. The velocity-independent part of the third term at the right-hand side of Eq. (1) is the radiation pressure force term. whereas the velocity-dependent parts are the drag terms. Solar wind drag is caused by the interaction between solar wind ions and the dust particle, and the net effect is similar to that of PR drag. The ratio of solar wind drag to PR drag is represented by $s w$ in Eq. (1). We have assumed $s w$ to be 0.35 based on à recent calculation (Gustafson 1994). The radiation pressure coefficient, $Q_{\mathrm{pr}}$, depends on the properties (density. shape, size, etc.) of the particle. Traditionally. a dimensionless quantity, $\beta$, is introduced to specify the effect of radiation pressure and PR drag. It is defined as

$$
\beta=\frac{\text { radiation pressure force }}{\text { solar gravitation force }}=\frac{S A Q_{\mathrm{pr}} r^{2}}{c G M m}
$$

In our numerical models, we study dust grains having $\beta$ equal to 0.4 . 0.2. 0.1 . and 0.05 . The corresponding sizes 
(diameters) for these four $\beta$ values are $1,2,4$, and $9 \mu \mathrm{m}$. respectively. The conversion from $\beta$ to size is based on the assumption that all particles are spherical "astronomical silicates" (Draine and Lee 1984) with $2.7 \mathrm{~g} \mathrm{~cm}^{-3}$ density and using Mie theory to integrate over the solar spectrum (Gustafson 1994). Particles smaller than $1 \mu \mathrm{m}$ usually do not remain in bound orbits once they leave their large parent bodies, because of radiation pressure. Particles larger than $9 \mu \mathrm{m}$ usually do remain in bound orbits and spiral toward the Sun under drag forces: however. due to the long integration times involved (it took approximately 10 months to complete our numerical simulation on 1- to 9- $\mu \mathrm{m}$ particles), we have not modeled the orbital evolution of particles larger than $9 \mu \mathrm{m}$. Our model serves as a first step to study small KB dust grains. The evolution of much larger dust grains remains a project for the future.

We assume dust grains are released (with zero relative velocity) from parent bodies located at $45 \mathrm{AU}$. with proper eccentricities and inclinations equal to 1$) .1$ and $10^{\circ}$. respectivelv. These are consistent with the ohservations and sta: Mtcal studes which rhow $\mathrm{KB}$ ohlects are in low-eccentricIty urouts with inclinations less than 12 (Jewitt and Luu 1995. Cochran et al. 1995). The initial forced elements are calculated by the secular perturbation theory (Brouwer and Clemence 1961) using the osculating orbital elements of seven planets. The initial proper longitudes of ascending node, proper longitudes of the pericenter. and mean longitudes of dust grains (before they left their parent bodies) are each randomly chosen between $0^{\circ}$ and $360^{\circ}$ to simulate their collisional origin. The forced and proper orbital elements are combined to form the initial osculating orbital elements of the parent bodies. When a dust grain leaves its parent body, it immediately "sees" a less massive Sun than does the parent body, due to solar radiation pressure on the particle. This changes the semimajor axis, eccentricity, and sometimes the longitude of pericenter of the dust grain. All these effects are included in our calculations.

The interaction between charged dust grains and the solar wind-associated magnetic field is not included in our model. The uncertainty involved in the structure. as well as the time variation, of the field makes it extremely difficult to model. At $1 \mathrm{AU}$, the Lorentz force, $F_{\mathrm{L}}$, on a typical $1-\mu \mathrm{m}$ dust grain. charged to $10 \mathrm{~V}$, is less than $2 \%$ of the gravitational force due to the Sun. $F_{\mathrm{g}}$. (Leinert and Grün 1990 ). For a $10-\mu \mathrm{m}$ grain, $F_{\mathrm{L}}$ is almost four orders of magnitude smaller than $F_{\mathrm{g}}$ at $1 \mathrm{AU}$. The ratio of $F_{\mathrm{L}}$ over $F_{\mathrm{g}}$ slowly increases with increasing heliocentric distance; however, as a dust grain moves around the Sun, solar rotation brings different sectors of the spiral structure of the magnetic field past the dust grain (e.g.. Schwenn 1990, Mariani and Neubauer 1990). Together with the fact that the polarity of the solar magnetic field changes on a 22-year cycle and the charge on a dust grain changes in an unknown fashion with time, the net effect of the solar magnetic field on a dust grain's orbital evolution may not be very significant. The actual answer. however. remains an open question.

The interactions between a charged dust grain and the magnetospheres of giant planets are also not included in our calculations. This is because (1) the solar wind pressure modifies outer planetary magnetospheres in an unpredictable way over time: (2) planetary dipole fields are not aligned with planetary spin axes and the spin rotation phases will be totally uncertain in our calculations: (3) like the Earth's magnetic field. planetary fields may change with time; and (4) dust grain charge in a planetary magnetosphere is uncertain. Ice grains and their associated evaporation are not considered in our model; we model only silicate dust grains of density $2.7 \mathrm{~g} \mathrm{~cm}^{-3}$.

For a given $\beta$, the equations of motion of seven planets. which gravitationally interact with one another, and 20 dust grains are integrated by the implicit Runge-Kutta integrator, RADAL (Everhart 1985). on an IBM ES 9000) supercomputer and an HP $9000715 / 75$ workstation. We follow the orbital evolution of each dust grain until it cither spirals into the sun or is clected from the solar stslem (due to gravitational scattering by planets).

\section{EVOLUTION OF DUST GRAINS}

The evolution in semimajor axis. eccentricity, and inclination of one of the $9-\mu \mathrm{m}$ dust grains is shown in Fig. 1. This dust grain started its journey from about $47.5 \mathrm{AU}$ (the effect of solar radiation pressure force increases the semimajor axis of a dust grain on its release from the large parent body). It passed through several exterior MMRs with Neptune before it was trapped in the $4: 3$ MMR with Neptune (at $35.2 \mathrm{AU}$ ) for about $3.6 \mathrm{myr}$. After it escaped this resonance, it was trapped briefly in the $1: 1 \mathrm{MMR}$ with Neptune (at 29.6 AU). It continued its journey toward the Sun after that and eventually was scattered out of the Solar System by Jupiter.

The next example is a $4-\mu \mathrm{m}$ dust grain (Fig. 2). This dust grain started its journey from about $50 \mathrm{AU}$. It was trapped in the $3: 2$ MMR with Neptune (at $38.1 \mathrm{AU}$ ) for about 16 myr. While trapped in this resonance, its eccentricity increased to about 0.37 before it escaped via a close encounter with Neptune. After it escaped the resonance via a close encounter with Neptune, it was trapped briefly in the 1:1 MMR with Uranus (at 18.6 AU). After it escaped the last resonance. this particle was able to continue its journey toward the Sun until it was removed from the Solar System by a close encounter with Jupiter.

The next example is a $2-\mu \mathrm{m}$ dust grain. It started its evolution from about $61 \mathrm{AU}$. due to radiation pressure. After passing several MMRs with Neptune, it was trapped in the 7:5 MMR with Neptune (at 35 AU) for about 1.8 myr (Fig. 3). After it escaped that resonance, it was able to pass by the four giant planets and made its journey all 

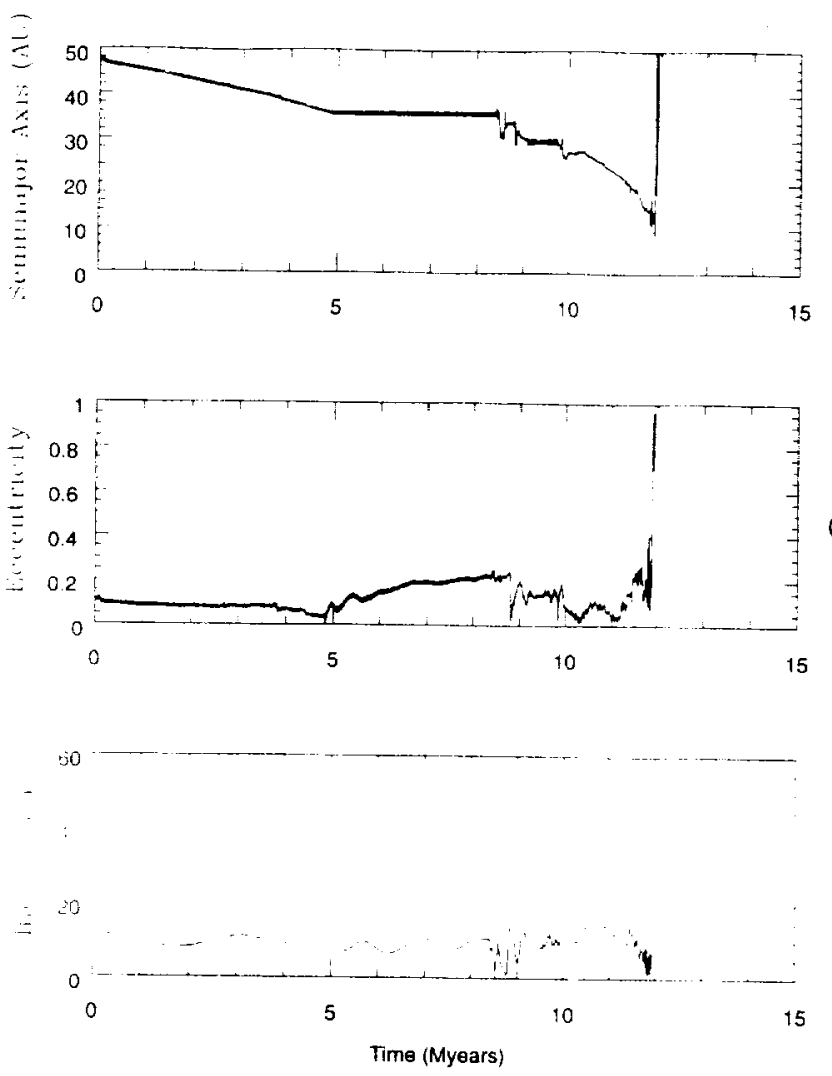

FIG. 1. Orbital evolution of a $9-\mu \mathrm{m} \mathrm{KB}$ dust grain. It started its journey from 47.5 AU due to the effect of radiation pressure. It was trapped in the $4: 3 \mathrm{MMR}$ with Neptune for about $3.6 \mathrm{myr}$ and was removed from the resonance before its eccentricity reached the possible maximum salue of 0.31 . It continued its journey afterward. and was trapped in the 1: 1 MMR with Neptune for about 650,000 years. Eventually this grain passed by Neptune. Uranus, and Saturn before it was ejected from the Solar System by Jupiter. To avoid possible confusion with dust grains that spiral into the Sun. we plot the semimajor axis of a grain as positive infinity when it becomes hyperbolic.

the way to the Sun. When this dust grain crossed the orbit of the Earth. its eccentricity was surprisingly small. about 0.08 . Its inclination was about $15^{\circ}$.

The next example is a $1-\mu \mathrm{m}$ dust grain (Fig. 4). The radiation pressure force pushes its semimajor axis to about $130 \mathrm{AU}$ after its release from the parent body. It was trapped in a 2:1 MMR with Neptune (at 40.3 AU) for about half a million years. After it escaped the resonance. it evolved all the way to the Sun. When this dust grain passed by the orbit of the Earth, its eccentricity and inclination were 0.132 and $16.6^{\circ}$, respectively.

Another example of evolution is shown in Fig. 5. It is a $f-\mu \mathrm{m}$ dust grain. This is one of the few grains in our samples that was trapped in two relatively long resonances. It started from about $51 \mathrm{AU}$ and was trapped in a 7:4 MMR with Neptune (at $42.2 \mathrm{AU}$ ) for about $6.1 \mathrm{mvr}$. After it escaped the resonance. $f t$ was trapped briefly in a $13: 10$
MMR with Neptune (at $34.6 \mathrm{AU}$ ) and then in a $1: 1 \mathrm{MMR}$ with Neptune (at $29.1 \mathrm{AU}$ ). Its final resonance trap was in a $1: 1 \mathrm{MMR}$ with Uranus for about $8.2 \mathrm{myr}$. In both long-duration resonances. the inclination of the dust grain tended to decrease with time. Based on previous work (Liou et al . 1995b), a secularly decreasing inclination might be expected. After it left the last resonance. it continued its journey toward the Sun until it was gravitationally scattered out of the Solar System by Saturn.

The effect of solar radiation pressure is to change the effective mass of the Sun as "seen" by dust grains. This changes the semimajor axis, eccentricity, and sometimes the longitude of perihelion of a dust grain on its release from the large parent body. It also modifies the location of a $p: q$ MMR with a planet. The new MMR location is given by

$$
a=a_{\mathrm{pl}}(1-\beta) \cdot\left[\frac{p}{q}\right]^{2 / 3} \equiv a_{\mathrm{n} 1}(1-\beta)^{1 / 3} K^{2 / 3} .
$$
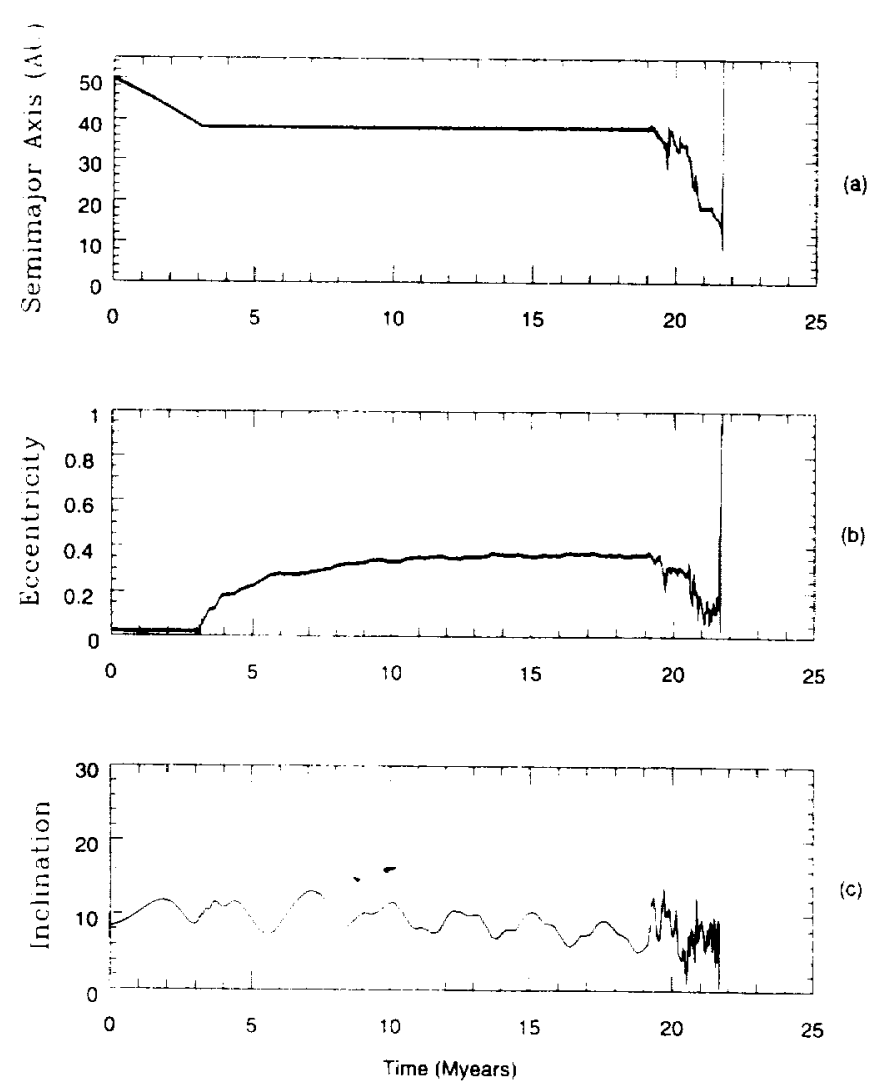

FIG. 2. Orbital evolution of 4- $\mu \mathrm{m} \mathrm{KB}$ dust grain. It began its evolution from about $50 \mathrm{AU}$. It was trapped in the $3: 2 \mathrm{MMR}$ with Neptune for about 16 myr. While trapped, its eccentricity increased to about 0.37 before being removed from the resonance by close encounters with Neptune. This 0.37 is consistent with the maximum eccentricity predicted by Eq. (4). It was later briefly trapped in the 1:1 MMR with Uranus and eventually ejected from the Solar System by Jupiter. 

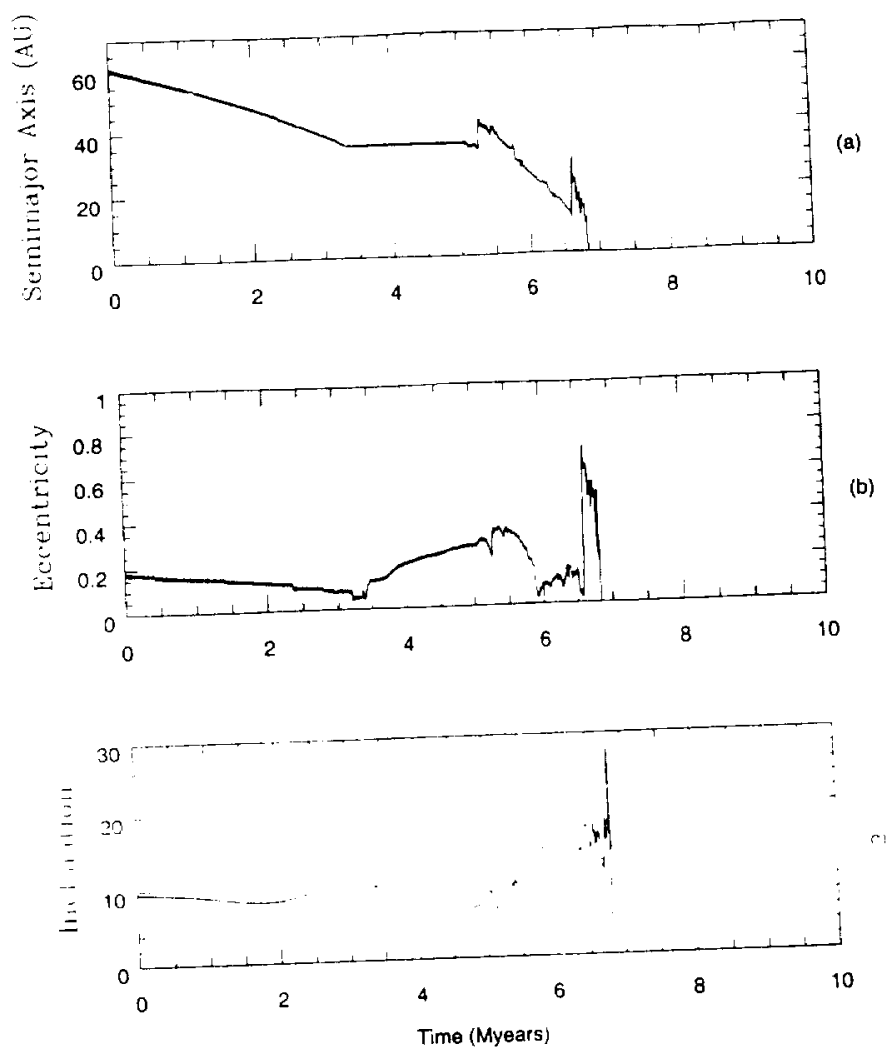

FIG. 3. Orbital evolution of a $2-\mu \mathrm{m} \mathrm{KB}$ dust grain. It was trapped in the 7:5 MMR with Neptune for about $1.8 \mathrm{myr}$. While trapped, its eccentricity increased to about 0.34 before being removed from the reso. eccentricity incre encounters with Neptune. The 0.34 is consistent with the maximum eccentricity predicted by $\mathrm{Eq}$. (4). After the grain escaped the resonance, it was able to pass by all the planets and completed its journey to the Sun. Even with the large variations in eccentricity and inclination over its evolution, this dust grain finally had a small eccentricity $(0.08)$ and inclination $\left(15^{\circ}\right)$ when it crossed the orbit of the Earth.

where $a_{\mathrm{pl}}$ is the semimajor axis of the planet, and $p$ and $q$ are two integers that specify a particular, $p: q$, resonance. The parameter $K$, defined as the ratio $p / q$, is larger than 1 for an exterior MMR. less than 1 for an interior MMR. and equal to 1 for a $1: 1 \mathrm{MMR}$.

In a Sun-dust two-body system. the effect of PR drag (and similarly solar wind drag) is to take away orbital energy and angular momentum of the dust grain. While the orbit of the grain circularizes and spirals toward the Sun, its inclination (with respect to an arbitrary reference plane) remains unchanged (e.g., Wyatt and Whipple 1950 , Burns et al., 1979). When a third body (planet) is involved, however, the evolution of the grain becomes more complicated. In addition to the short-period oscillatory as well as the secular perturbations from the planet (e.g., Brouwer and Clemence 1961), two important dynamical effects are brought into the system. They are the MMR perturbations by the planet as well as single-event gravitational scattering by the planet. A dust grain's spiral motion toward the Sun can be interrupted by getting trapped into one or more MMRs with that planet. In a planar circular Sun-planetdust three-body system. if the drag rate is small and the planet is massive enough such that the evolution of the grain is in the adiabatic regime, the capture probability into a given exterior MMR can be estimated (e.g., Henrard 1982. Beaugé and Ferraz-Mello 1994). Otherwise. numerical integration of the equations of motion of the system has to be performed. As $\beta$ increases, dust grains tend to become trapped in exterior resonances closer to the planet (Šidlichovský and Nesvorný 1994). Once the grain is trapped in an exterior resonance, its eccentricity increases and may eventually reach a maximum value given by

$$
\frac{2+3 e}{2\left(1-e^{2}\right)^{3 / 2}}=K
$$

This expression gives the value of the eccentricity required hy the condition of a quasi-stable trap (Beauge and FerrazMello 1994. sidichorsky and Vesvorny 1994. Liou e" at.
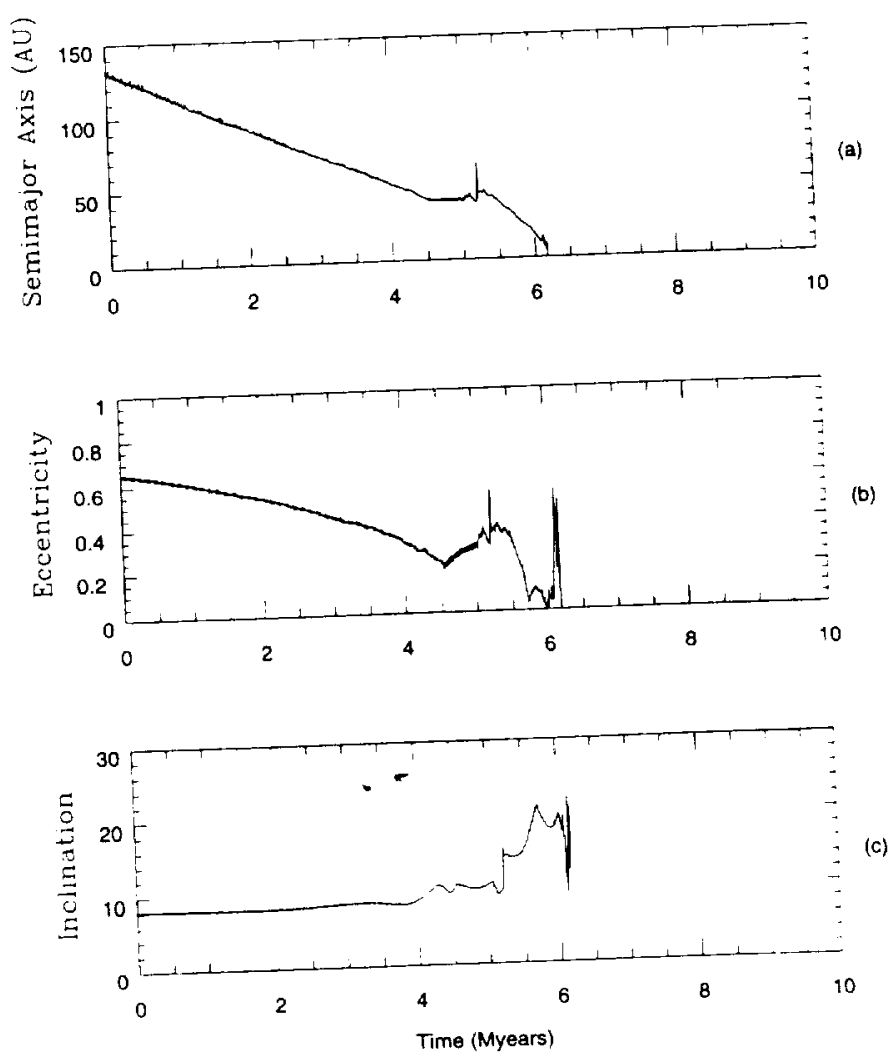

FIG. 4. Orbital evolution of a $1-\mu \mathrm{m} \mathrm{KB}$ dust grain. It started from 30 AU and was trapped in a 2:1 MMR with Neptune for half a million After it escaped the resonance, this grain evolved all the way to the Sun It eccentricity and inclination were 0.132 and $16.6^{\circ}$ when it passed by the Earth. 

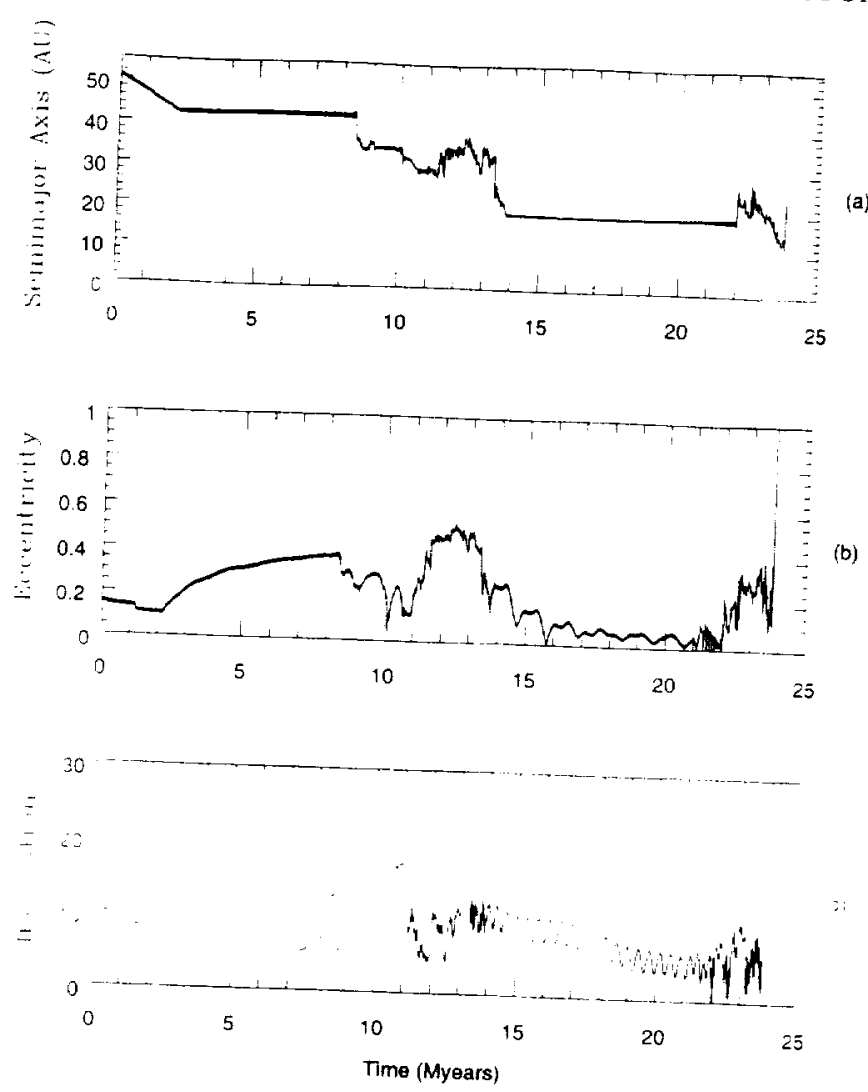

FIG. 5. Evolution of a $4-\mu \mathrm{m} \mathrm{KB}$ dust grain that was trapped in two long resonances: a $7: 4$ with Neptune and a $1: 1$ with Uranus. While secular. though resonances. the inclination of the grain went through secular. though oscillatory, decreases due to PR and solar wind drag.

1995b): however, due to the unstable nature of the exterior MMR trap with PR drag (Gomes 1995), dust grains eventually escape the resonance via an ever-increasing amplitude of the resonance angle oscillation and. finally, via close encounters with the planet. Afterward, they cither continue their journey toward the Sun or leave the Solar System on hyperbolic orbits. If a dust grain is trapped in a 1:1 MMR with the planet, its Jacobi "constant" (when there is no drag, this is a constant of the motion in the restricted three-body problem, e.g., see Brouwer and Clemence 1961) increases with time, which forces the orbit to expand from a tadpole to a horseshoe (in the rotating reference frame), and. finally, close encounters with the planet eventually remove the dust grain from the resonance (Liou et al. 1995b). For any dust grain that becomes trapped into an interior MMR with a planet. trapping is always unstable and temporary (Liou et al. 1995b).

In a three-dimensional Sun-planet-dust system. where the orbit of the dust grain is inclined with respect to the orbital plane of the planet. the dynamics become somewhat complicated. The inclination of a dust grain trapped in an MMR tends to be damped due to PR and solar wind drag (Liou et al. 1995b); however, secular resonances (when more than one planet is involved) and gravitational scatter ings with the planet can also change the orbital inclination of the dust grain. They may well dominate drag effects and increase inclination. High inclinations will increase the encounter velocities of $\mathrm{KB}$ dust grains with the Earth, should they make their journey all the way to the inner Solar System. This is an important tactor when isidering the possible contribution of the KB dust gi ins to the collected IDPs. We discuss this in more detail in Section 6.

The real Solar System, with nine planets, is more complicated than a simple three-body system: nevertheless, when a dust grain is trapped in an MMR with a planet, the dominant perturbation, besides the Sun's influence, is due to that planet. A three-body approach to understand the dynamics involved is a good approximation. The evolution of dust grains in our numerical simulations are in qualititive agreement with theory. While trapped in exterior MMRs. the dust grain's eccentricity increases to about the predicted maximum value given hy Eq. (4), unless close encounters with the trapping planet interrupt the trapping process. or another planet watso in some resonance whth the gran. The orbital inclination ot a dust grain in a long resonance can be seen to go through a large short-term variation added to a slow secular decrease when a secular resonance is not involved.

An unusual evolution of the KB dust grain is shown in Fig. 6. This is a $9-\mu \mathrm{m}$ dust grain. Shortly after it started its journey from $48 \mathrm{AU}$, it was trapped in a 7:4 MMR with Neptune (at $42.97 \mathrm{AU}$ ) for nearly 150 myr. While it was trapped, its inclination increased from about $10^{\circ}$ to more than $40^{\circ}$. This is an example of how a secular resonance can change the inclination of an orbit. At $42.97 \mathrm{AU}$, the precession rate of the longitude of the ascending node of the dust grain is half the eigenfrequency of the system that controls the precession rate of the proper longitude of the ascending node of Neptune. Its eccentricity increased slowly but had not reached the maximum value 0.43 given by $\mathrm{Eq}$. (4). The high inclination may be responsible for such an unusually long trap.

\section{STATISTICAL DESCRIPTION OF DUST GRAINS COMING FROM THE KUIPER BELT}

In our numerical calculations, we calculated the orbital evolution of 80 different KB dust grains: there were 20 grains at each grain diameter of 1.2. 4, and $9 \mu \mathrm{m}$. This covers approximately three orders of magnitude in terms of the particle masses. Surprisingly, their overall evolution patterns are very similar. Most of the grains are trapped for millions of years in one of the exterior MMRs with Neptune or Uranus. When they escape the resonances, they are nearly always able to pass by Neptune and Uranus and continue their journey toward the Sun. Only three dust grains out of the total of 80 samples were ejected 

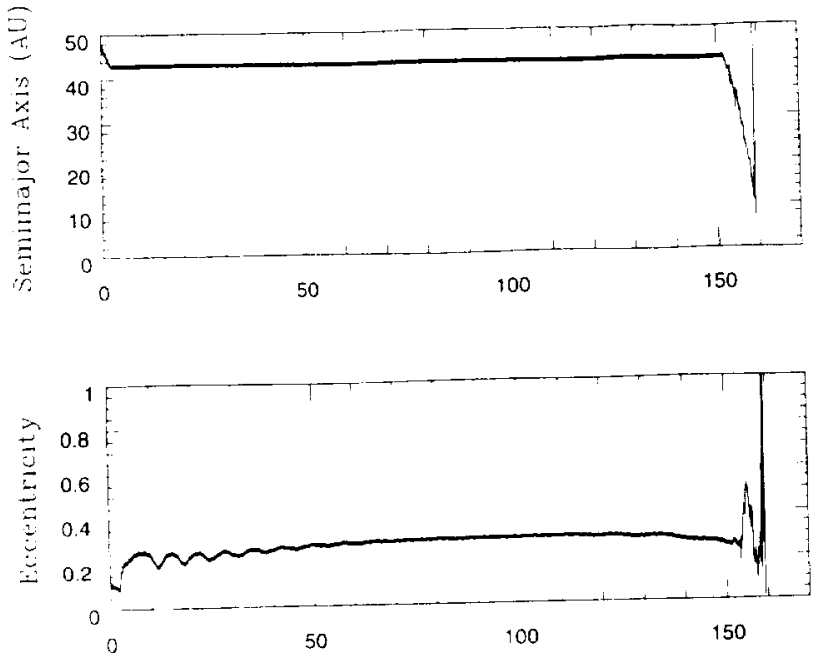

(b)

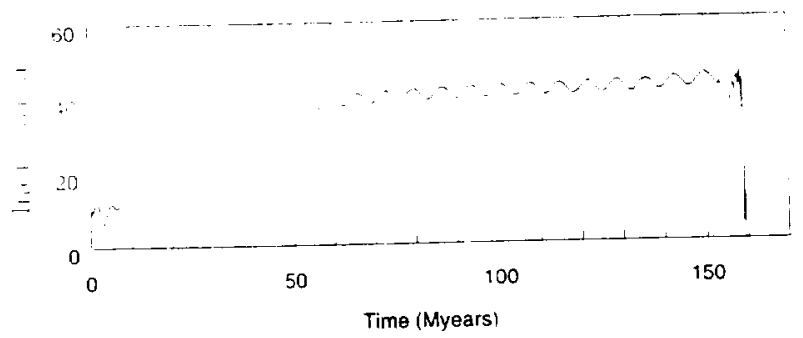

FIG. 6. Lnusual evolution of a $9-\mu \mathrm{m} \mathrm{KB}$ dust grain. It was trapped in the 7:4 MMR with Neptune for nearly $150 \mathrm{myr}$. At the resonance location, the precession rate of the longitude of ascending node of the dust grain is two times slower than the proper precession rate of Neptune's orbital plane. This secular resonance pumped up the inclination of the grain to more than $40^{\circ}$. and overwhelmed drag forces that tend to decrease inclination.

from the Solar System by Neptune. Trapping intervals due to Jupiter and Saturn are short, by comparison. The statistics are shown in Fig. 7.

The consequence of dust grains being trapped in exterior MMRs with Neptune (or with Uranus) is the formation of dust rings like the ring of dust of asteroidal origin near the Earth's orbit (Jackson and Zook 1989, Dermott et al. 1994b, Reach et al. 1995). Because of their large eccentricities, these rings spread out over quite large ranges, approximately from 20 to $40 \mathrm{AU}$. With their large distances from the Sun and Earth. it is highly unlikely that these rings are telescopically detectable.

After dust grains pass by Uranus. gravitational scatterings due to Saturn and Jupiter dominate their final destiny. Jupiter and Saturn do not appear to be able to trap dust grains in their MMRs in quasi-stable orbits inside $20 \mathrm{AU}$. None of the sample grains were trapped longer than 500,000 years with either planet. The large masses of Jupiter and Saturn. however. make their gravitational scatterings more effective than those of Neptune and Uranus (e.g. Öpik 1963. Dohnanyi 1978). About three-quarters of all sample dust grains were ejected from the Solar System by either Saturn or Jupiter. Thus, these two major planets are primarily responsible for preventing most (75\%) of the KB dust grains from evolving in toward the inner part of the Solar System.

About $20 \%$ of the sample dust grains in each size range was able to pass by Saturn and Jupiter. Once dust grains passed by Jupiter. they evolved all the way to the Sun. None of the dust grains were ejected from the Solar System by any terrestrial planets.

The KB dust grains that do enter the inner Solar System (inside Jupiter) have small eccentricities and inclinations when they cross the orbit of the Earth. Figure 8 shows their eccentricities and inclinations when their semimajor axes reach $1 \mathrm{AU}$. Since PR and solar wind drag reduce the eccentricities of dust grains as they spiral from the outer Solar System toward the Sun we would expect them to have small eccentricities. when no planetary perturbatoons are considered. as they approach the Farth. When the - sunances and stavtlational catternos that dramattails affect the dust grains eccentricities and inclinations throughout their orbital evolution (Figs. 1-6) are considered, however. it is quite unexpected that they approach the Earth with asteroidal-type orbits. The low eccentricities

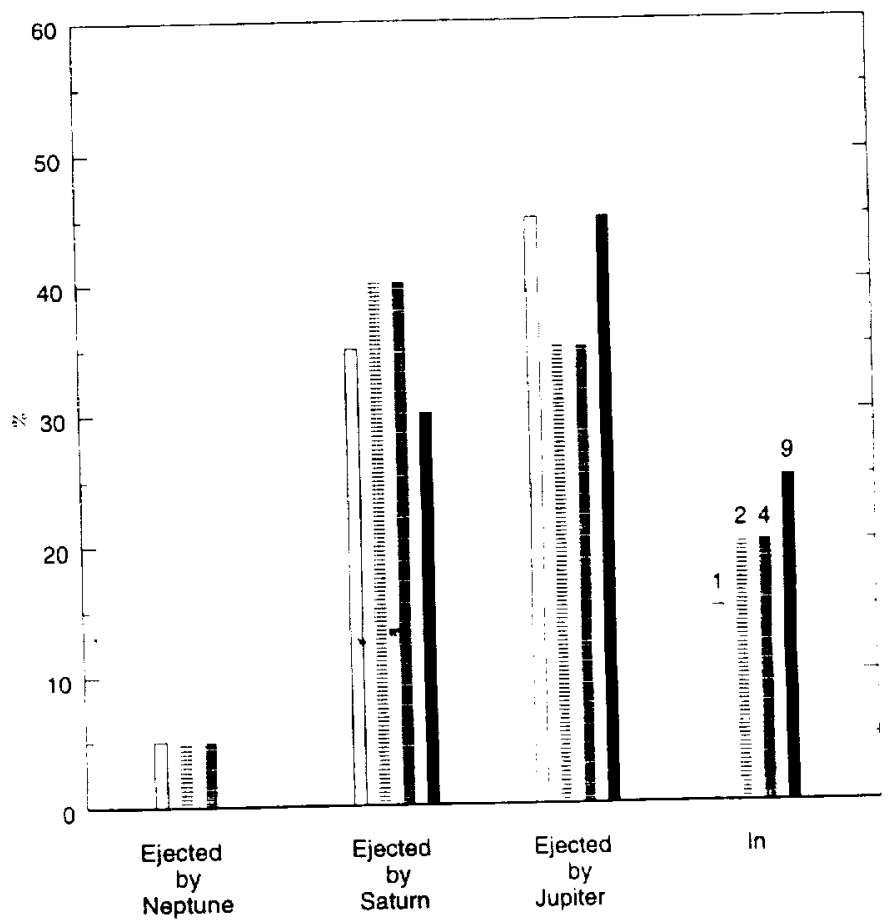

FIG. 7. Summary of the evolution of KB dust grains. Even though their sizes are different (1 $109 \mu \mathrm{m}$ in diameter. labeled above the right histogram). the overall patterns are very similar. Most of the dust grains were ejected from the Solar System by Jupiter or Saturn. Ahout $20 \%$ of the grains were able to evolve all the way to the Sun 


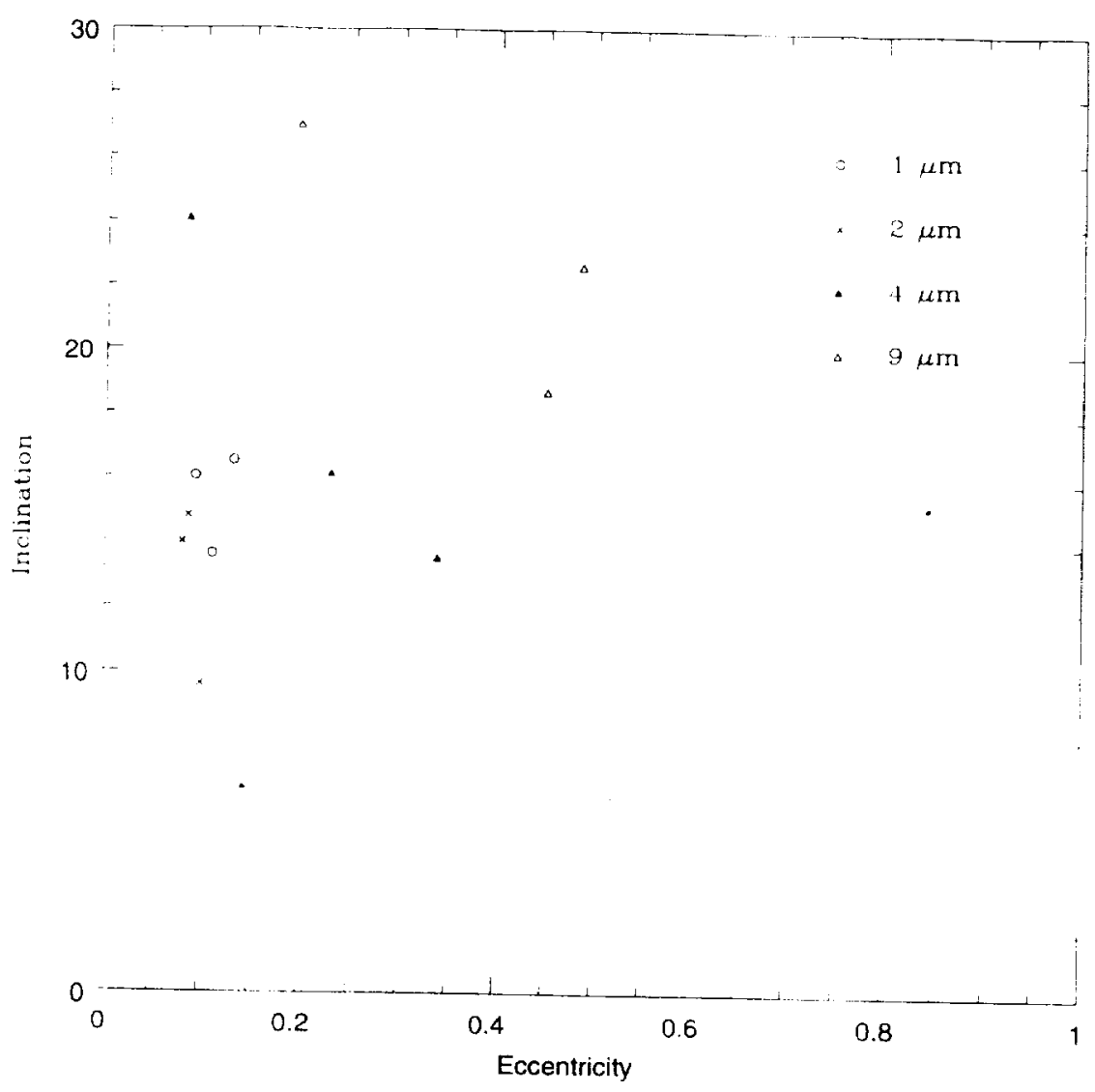

FIG. 8. Distributions in eccentricty and inclination at 1 AU 1 of the KB dust grains that enter the inner Solar System. For small $11-t \mu m$ grains.
their eccentricities and inclinations are similar to those of the asteroidal dust grains.

and inclinations of the Earth approaching $\mathrm{KB}$ dust grains mean they are more likely to be captured by the Earth and to survive atmospheric entry than most cometary dust grains. After these dust grains passed by the Earth. they continued their journey toward the Sun. Most of them had eccentricities smaller than 0.05 when they were within 0.2 AU from the Sun. Their inclinations remained approximately the same as those shown in Fig. 8 .

\section{COLLISIONS BETWEEN INTERPLANETARY DUST GRAINS AND WITH INTERSTELLAR DUST}

In our numerical calculations. mutual collisions between dust grains and collisions with interstellar dust particles were not included: however. because of the long evolutionary lifetime of the KB dust grains. collisions may destrov some dust grains and interrupt their orbital evolution. We shall see that collisions may prevent particles larger than a certain size from evolving toward the inner Solar System even though, from the dynamical point of view, such particles are capable of evolving all the way to the Sun.

It is difficult to calculate the real collisional destruction of $\mathrm{KB}$ grains due to intérstellar dust grains. This is due primarily to our limited knowledge of interstellar dust (e.g.. its spatial distribution and how it varies with time). In the following we estimate the role of collisions based on the recent Llyses measurement and on a few simple assumplions.

In 1992 . the spacecraft Ulysses discovered interstellar dust grains while passing the vicinity of Jupiter (Grün et al. 19931. At 5 AU, the mean flux is $8 \times 10^{-5} \mathrm{~m}^{2} \mathrm{sec}^{-1}$. The masses of those particles range from $10^{-15}$ to $5 \times 10^{-12}$ $\mathrm{g}$ with a mean mass of $8 \times 10^{-1.3} \mathrm{~g}$ and they travel in excess of $26 \mathrm{~km} \mathrm{sec} \mathrm{'} \mathrm{(Grün} \mathrm{et} \mathrm{al.} \mathrm{1993,} \mathrm{see} \mathrm{also} \mathrm{Baguhl} \mathrm{et} \mathrm{al.}$ 1995). According to Eq. (14) in Grün et al. (1985). all but the smallest are easily capable of collisionally shattering 9- $\mu \mathrm{m} \mathrm{KB}$ dust grains. For the purpose of our estimate. we alssume th: mean flux at $5 \mathrm{AU}$ is the same throughout the Solar Syictem and that it does not vary with time. From the mean thux, the average time for one collision. $t_{6}$. for a spherical KB grain with diameter $d \mu \mathrm{m}$ to encounter an interstellar dust grain. with diameter $d_{i} \mu \mathrm{m}$. is

$$
t_{\mathrm{c}}=\frac{504}{\left(d+d d_{\mathrm{i}}\right)^{2}} 10^{\mathrm{h}} \text { years }
$$


If we take the mean interstellar dust particle mass to be $8 \times 10^{-13} \mathrm{~g}$, the mean diameter for such spherical particle with density $1 \mathrm{~g} \mathrm{~cm}^{-3}$ is $1.2 \mu \mathrm{m}$. For $\mathrm{KB}$ grains with diameters $1,2.4$. and $9 \mu \mathrm{m}$, the $t_{\mathrm{c}}$ are $104,49.19$. and $4.8 \mathrm{myr}$. respectively.

Mutual collisions between Kuiper belt dust grains can also affect their evolution. But the lack of knowledge of their size and spatial density distributions in the region of $\mathrm{KB}$ and the changes of these distributions as differentsized grains evolve differently toward the Sun (different starting locations, different drag rates, different resonance trapping. etc.) all make collision rate calculations very uncertain. Here we simply estimate the mutual collision lifetime of a dust grain in interplanetary space with grains of the same size. The average penetration flux for meteoroids larger than $8.3 \times 10^{-11} \mathrm{~g}$ measured by Pioneer 10 between 5 and $18 \mathrm{AU}$ is about $4 \times 10^{-\mathrm{h}} \mathrm{m}=\mathrm{sec}^{-1}$ (Humes 1980). The mass threshold corresponds to an $8.4-\mu \mathrm{m}$-diameter spherical grain with the same physical conditions as those of the dust grains in our calculation. If we assume that this constant tlux is the same exen hevond: Al and that the meteoroids are of the same size. $9 \mu \mathrm{m}$. then the average time for one dust grain to have a collision with another one is

$$
\tau_{\mathrm{c}}=\frac{2523}{(9)^{2}} 10^{6} \text { years }=31.1 \times 10^{\circ} \text { years }
$$

The time scale of Eq. (6) is longer than the one given by Eq. (5) for a $9-\mu \mathrm{m}$ dust grain colliding with an interstellar grain. When interplanetary dust grains of smaller size (with the lower boundary at $1 \mu \mathrm{m}$ ) are included, $\tau_{\mathrm{c}}$ is expected to be shortened: however. all KB dust grains are in prograde orbits; the impact velocity between them may not be high enough to always result in a catastrophic collision. Therefore, mutual collisions between $\mathrm{KB}$ dust grains appear to be not as important as collisions with interstellar dust grains for the $9-\mu \mathrm{m}$ dust grains. It is likely true for other sizes also. Based on a previous study of the collisional balance of the meteoritic complex, which includes the effects of PR drag and the observed size distribution of micrometeoroids down to $10^{-15} \mathrm{~g}$ near $1 \mathrm{AU}$ (Grün et al. 1985), dust grains of 1 to $9-\mu \mathrm{m}$ have similar collisional lifetimes in such a complex. Whether or not these relative collision lifetimes remain valid in the outer Solar System remains an open question.

About $90 \%$ of asteroidal dust grains smaller than $10 \mu \mathrm{m}$ in diameter evolve from $3 \mathrm{AU}$ to the Earth without being affected by collisions due to the interplanetary dust complex (Gustafson 1994). Therefore. collisional destruction due to the addition of asteroidal dust grains does not seem to affect $\mathrm{KB}$ dust grains smaller than $10 \mu \mathrm{m}$ in the inner Solar System.

The actual orbital evolution lifetimes of particles of dif-

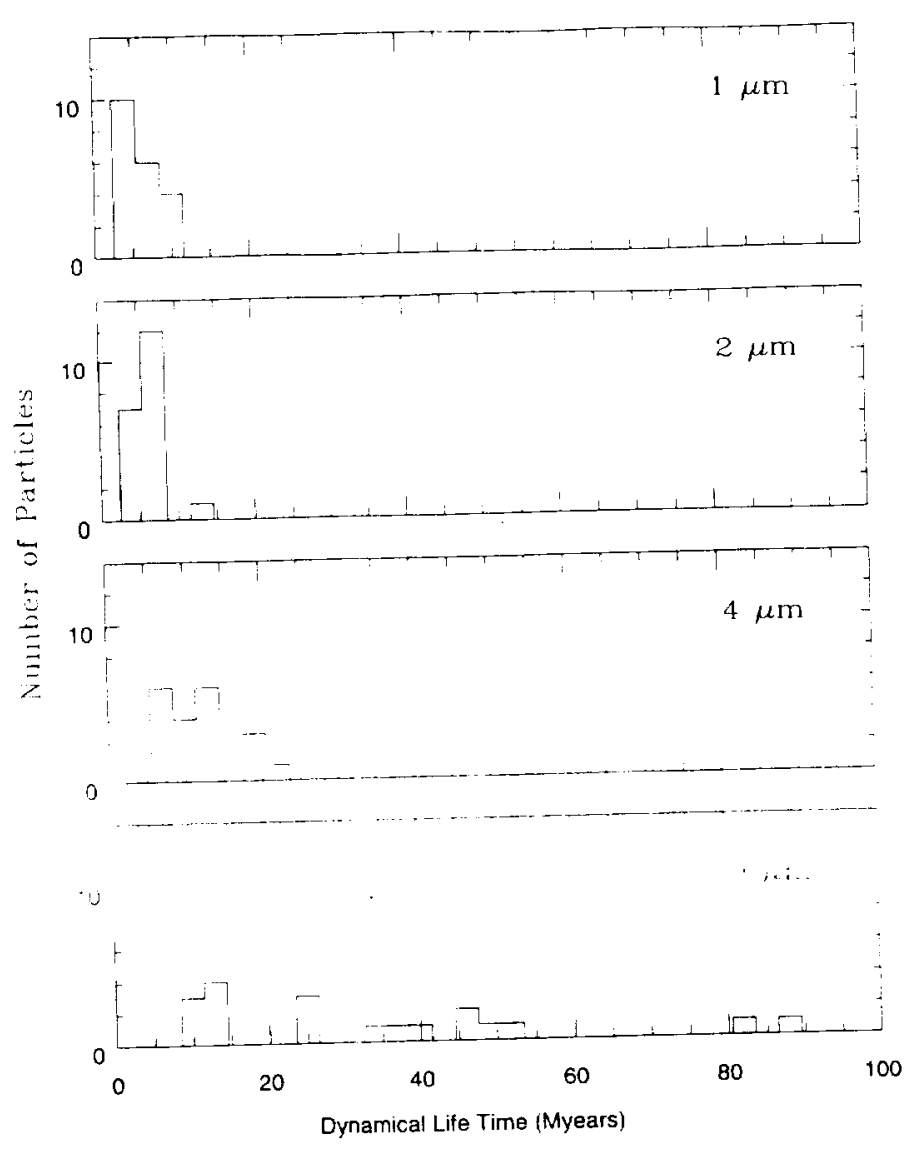

FIG.9. Dynamical lifetime (from when they were released from their parent bodies to the time they were ejected from the Solar System or spiraled into the Sun) of the KB dust grains. Several factors determine the dynamical lifetime of a KB grain. They are. how large its semimajor axis became after it was released from its parent body. its drag rate. resonance trappings, and gravitational scattering by planets. The $9-\mu \mathrm{m}$ grain from Fig. 6. which has a lifetime of 150 myr. is not included here.

ferent sizes from our numerical integrations are shown in Fig. 9. Several factors determine the evolution lifetime of a grain. They include the starting semimajor axis of the grain (a function of $\beta$ and where the dust grain was released from its parent body s orbit). the drag rate. resonance trapping, and gravitational scattering by planets. Based on our collision estimates. it appears that collisions due to interstellar dust grains and mutual collisions have little effect on the orbital evolution of 1-. 2-. and $4-\mu \mathrm{m} \mathrm{KB}$ dust grains: however. collisions seem likely to destroy most grains around $9 \mu \mathrm{m}$ before they complete their orbital evolution under the PR and solar wind drag. The interstellar dust grains detected by Ulysses are capable of causing catastrophic destruction to dust grains up to about $50 \mu \mathrm{m}$ in diameter (Grün et al. 1985). For even larger KB dust grains the measured interstellar dust grains appear too small to cause catastrophic destruction in any one single impact. even though "many small projectile impacts may 
eventually disrupt the target particle although an individual impact would not result in complete rupture " (Grün et al. 1985). Also, as the size distribution of interstellar grains is not yet well determined. the flux of large interstellar grains is rather uncertain. Whether or not these larger KB dust grains can survive collisional destruction and pass by giant planets remains an open question.

\section{KUIPER BELT GRAINS AS A SOURCE OF ZODIACAL LIGHT AND IDPS}

Comets and asteroids have long been considered the two major sources that deposit dust particles into interplanetary space which. in turn. give rise to the zodiacal light. From the in situ spacecraft experiments. it has been shown that the main contribution to the zodiacal light is from particles that range from $2^{1} 10200 \mu \mathrm{m}$ in diameter (Grün "t al. 1985). On the other nand. from the Infrared Astronomical Satellite IIRASi inservations at $25-\mu \mathrm{m}$ wavekength and from donamica. modeling. 11 was bown that"

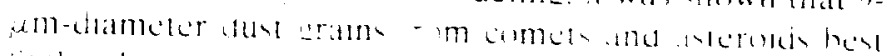
tit the observatwonat hape the andiatial light as a luncton of ecliptic longitudes (Dermott "t at. 199.ta. Liou et at. 1995a). In regard to the first model, it is not clear whether KB dust grains larger than $50 \mu \mathrm{m}$ can evolve to the inner Solar System. In regard to the second model. it appears that $\mathrm{KB}$ dust grains probably do not contribute significantly to the zodiacal light: however. this is based on our collision model which assumes a uniform (does not vary with distance from the Sun) and stable (does not vary with time) interstellar dust grain flux. In reality the spatial density of the interstellar dust grains is certain to vary as the Solar System moves around our galaxy. It is possible that at a time when the Solar System passes through a lower-density (lower than what Ulysses detected) interstellar dust cloud. $\mathrm{KB}$ dust grains larger than $+\mu \mathrm{m}$ may be able to evolve all the way toward the Sun and be a significant part of the zodiacal light.

Interplanetary dust particles collected by high-altitude aircraft from the stratosphere contain several distinctive types of primitive materials 1 e.g. Bradley et al. 1988. Clemett et al. 1993). These IDPs range from 1 to $50 \mu \mathrm{m}$ in diameters. They could be responsible for bringing the first organic materials to the Earth and contribute to the origin of life. Again. asteroids and comets are thought to be the two major sources for these IDPs (e.g.. Bradley et al. 1988): however. we argue here that $\mathrm{KB}$ dust grains with diameters around 1 to $4 \mu \mathrm{m}$ could te a significant portion of the small (between 1 and $4 \mu \mathrm{m}$ i collected IDPs.

Short-period comets are thought to come from the region of the KB (e.g.. Duncan et al. 1988. Quinn et al. 1990. Levison and Duncan 19931. They are KB objects that were perturbed by Neptune and thrown into the inner part of the Solar System. They čn bring in primitive materials from the outer Solar System: however, their mean orbital eccentricity and inclination are about 0.5 and $10^{\circ}$. respectively. Small dust grains released from these short-period comets are likely to have eccentricities larger than 0.5 . if they are still in bound orbits. Because of their high eccentricities. their inclinations will be pumped up while spiraling toward the Sun (Liou 1993). In general, this leads to a higher Earth-approaching encounter velocity (larger than $10 \mathrm{~km} \mathrm{sec}$. Jackson and Zook 1992), although there are a few exceptions (Jackson and Zook 1992). There is little gravitational focus enhancement for those with high Earth-approaching velocities. It is also difficult for them to survive the atmospheric entry heating thereby to fall down to the surface of the Earth or to be available for capture by high-altitude airplanes (Flynn 1989).

Small dust grains coming from long-period comets may not constitute a significant part of the collected IDPs. This is because long-period comets have highly eccentric urbits with random inclinations. The gain in total energy hs mall dust grans when they are released trom lonepertice som-

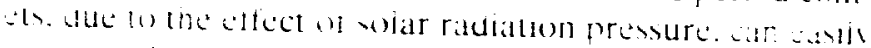
increase their orhital energy to such anl extent that eney leave the Solar System on hyperbolic orbits shortly atter leaving their parent bodies. For those that remain in bound orbits. the highly inclined orbits (including retrograde orbits) usually result in a very high Earth-approaching velocity.

Large dust grains coming from the main asteroidal belt approach the Earth with typical eccentricities less than 0.2 and inclinations similar to those of their parent bodies (with a mean around $10^{\circ}$ ). This typically results in a low (less than $10 \mathrm{~km} \mathrm{sec}^{-1}$ ) Earth-approaching velocity / Jackson and Zook 1992). Small asteroidal dust grains have similar eccentricities and inclinations near the Earth ( Liou and Zook 1995). The gravitational focusing effect enhances their flux near the Earth (e.g., Dohnanyi 1978). A low Earth-approaching velocity also makes it easier for them than for highly eccentric orbit grains to survive the atmospheric entry heating. They are certainly a source of the collected IDPs: however. they only bring in materials from as far as about $4 \mathrm{AU}$ from the Sun. Whether primitive/ organic materials at that distance could survive the early Solar System T-Tauri wind and heating from the Sun is not clear.

When one compares the KB dust grains with either cometary or asteroidal dust grains. it becomes obvious that KB grains have certain adiantages to make up the carbonaceous unaltered small IDPs on Earth. They approach the Earth with eccentricities and inclinations similar to those of the asteroidal dust grains. The consequent low Earthapproaching velocities not only enhance their flux but favor their chances of surviving the atmospheric entry heating. They are likely to bring in primitive/organic materials from the outer Solar System. where primitive/organic materials 
should be better preserved simply because of their greater distance from the Sun. They could be a significant fraction of the small (less than $5-\mu \mathrm{m}$-diameter) collected IDPs. They could even be a source of the earliest organic material on the Earth that gave rise to life.

Is it possible to distinguish KB dust grains from other dust grains in the collected IDPs? If so, we can analyze them and learn more about material composition in the $\mathrm{KB}$ and increase our knowledge of the early Solar System. From a dynamical point of view, KB dust grains that cross the Earth's orbit appear just like asteroidal dust grains. with similar eccentricities and inclinations when they cross the orbit of the Earth. This implies that the atmospheric entry heating is similar for both types of grains. The major difference between KB and asteroid dust grains is their space exposure time. The solar flare track density measurement (e.g.. Bradley et al. 1984. Sandford 1986) may show a difference between them. KB dust grains should have a much higher track density than asteroidal dust grains. This suggests that among small collected IDPs. those with the honer whar hate track densils mat some trom the KB.

\section{CONCLUSION}

We have completed a study of the orbital evolution of micron-sized dust grains ( 1 to $9 \mu \mathrm{m}$ in diameter) coming from the region of the KB. Our model includes the gravitational forces of the Sun and planets, as well as solar radiation pressure and PR and solar wind drag on a dust grain. Interactions with solar magnetic fields and the magnetospheres of giant planets are not included in the calculation. The results show that a significant portion (about 20\%) of these dust grains are capable of evolving toward the inner part of the Solar System. With the continuous discoveries of large objects in the KB, the outer Solar System (from 30 to $50 \mathrm{AU}$ and beyond) has become a place that could potentially supply a large amount of dust grains to interplanetary space. Collisional destruction due to interstellar dust grains. however, may limit the significance of $\mathrm{KB}$ dust grains in contributing to the zodiacal light. Small KB dust grains (less than $9 \mu \mathrm{m}$ ) can evolve toward the inner Solar System without being seriously affected by collisions. For much larger KB dust grains (larger than about $50 \mu \mathrm{m}$ ). it remains a possibility that they may also enter the inner Solar System. To understand their exact contribution to the zodiacal light and in the collected IDPs. and to possibly distinguish them from other dust grains in the collected IDPs. more work is needed on the dust production rate in the KB. the chemistry in the outer Solar System. as well as numerical modeling of evolution of larger $\mathrm{KB}$ dust grains.

\section{ACKNOWLEDGMENTS}

We thank D. Jewitt and S. A. Stern for sending us preprints of their papers. We thank D. Hamilton for suggestions on the collision model.
Thanks also to H. F. Levison and an anonymous referee for their review comments. This research was performed while J.C.L. held a National Research Council-NASA/JSC Research Associateship.

\section{REFERENCES}

Backman. D. E.. A. Dasgupita, and R. E. Stencel 1995. Model of a Kuiper belt small grain population and resulting far-infrared emission. Astrophys. J. 450. L.35-L.38.

Baguhl, M. D. P. Hamilton. E. Grün. S. F. Dermott. H. Fechtig. M. S. Haniner, J. Kissel. B.-A. Lindblad, D. Linkert, G. Linkert, 1. Mann. J. A. M. McDonnell, G. E. Morfill. C. Polanskey, R. Riemann. G. Schwehm. P. Staubach. and H. A. Zook 1995. Dust measurements at high ecliptic latitudes. Science 268, 1016-1019.

Beaugé. C. And S. Ferraz-Mello 1994. Capture in exterior meanmotion resonances due to Poynting-Robertson drag. Icarus 110, $239-260$.

Bradley. J. P.. D. E. Brownlee, and P. Fraindorf 1984. Discovery of nuclear tracks in interplanetary dust. Science 226, 1432-1434.

BR ADLFYY.J. P., S. A. SANDFORD. AND R. M. WALKER 1988. Interplanetary dust particles. In Meteortes and the Early Solar System (J. F. Kerridge and M. S. Matthews. Eds. p. pp. $861-x 95$. Lniv of Arizona Press. Tueson

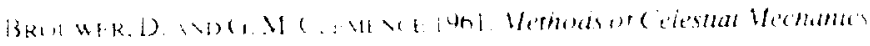
Academic Press. Vien York

Bi Rus. J. A.. P. L. L.MYY Aw S. Soter 1979. Radiation forces on small particles in the Solar System. larus 40, 1-48.

Clemetr. S. J.. C. R. Maechling, R. N. Zare, P. D. Swan, and R. M. WALKER 1993. Identification of complex aromatic molecules in individual interplanetary dust particles. Science 262. 721-725.

Cochran, A. L.. H. F. Levison, S. A. Stern, and M. J. Duncan 1995. The discovery of Halley-sized Kuiper belt objects using the Hubble Space Telescope. Astrophys. J. 455, 342-346.

Dermott, S. F., D. D. Durda, B. A. S. Gustafson, S. Jayaraman, J. C. Liou, AND Y. L. Xu 1994a. Zodiacal dust bands. In Asteroids, Comets, Meteors 1993 (A. Milani, M. Martini and A. Cellino, Eds.), pp. 127-142. Kluwer. Dordrecht.

Dermott, S. F., S. Jayaraman, Y. L. Xu, B. Å. S. Gustafson, and J. C. Lioc 1994b. A circumsolar ring of asteroidal dust in resonant lock with the Earth. Nature 369, 719-723.

Dohnanyı. J. S. 1978. Particle dynamics. In Cosmic Dust (J. A. M. McDonnell. Ed.), pp. 527-605. Wiley. Chichester

Draine. B. T. AND H. M. LeE 1984. Optical properties of interstellar graphite and silicate grains. Astrophys. J. 285, 89-108.

Duncan, M.. T. Quinn. And S. Tremaine 1988. The origin of shortperiod comets. Astrophys. J. 328, L69-L73.

Everhart, E. 1985. An efficient integrator that uses Gauss-Radau spacings. In Dinamics of Comets:-Their Origin and Evolution (A. Carusi and G. B. Valsecchi. Eds.). pp. 185-202. Reidel, Boston.

FLYNN, G. J. 1989. Atmospheric entry heating: A criterion to distinguish between asteroidal and cometary sources of interplanetary dust. Karus 77, $287-310$.

FLyNN. G. J. 1994. Does the Kuiper belt contribute significantly to the zodiacal cloud and the stratospheric interplanetary dust? Proc. Lunar Planet. Sci. Conf. 25rh. 379-380.

Gomes, R. S. 1995. The effect of nonconservative forces on resonance lock: Stability and instability. Icarus 115, 47-59.

Grün E., H. A. Zook. M. Baguhl, A. Balogh, S. J. Bame, H. Fechtig, R. Forsyibl. M. S. Haviter. M. Horanyi, J. Kissel, B.-A. Lindblad. D. Linkeri. G. Linkert. I. Mann. J. A. M. MCDonnell. G. E. Mor fill. J. L. Phillips. C. Polanskiy. G. Schwehm. N. Siddolif, P. 
Staubach. J. Svestka, and A. Taylor 1993. Discovery of jovian dust streams and interstellar grains by the Ulysses spacecraft. Nature 362, 428-430.

Grun, E. H. A. Zook, H. Ferhtig, and R. H. Giese 1985. Collisional balance of the meteoritic complex. Icarus 62. 244-272.

Gustafson. B. À. S. 1994 Physics of zodiacal cloud. Annu. Rev. Earth Planet. Sci. 22, 553-595.

Henrard, J. 1982. Captur : nto resonance: An extension of the use of the adiabatic invariants. lest. Hech. Dynam. Astron. 27, 3-22.

Humes, D. H. 1980. Results ol Pioneer 10 and 11 meteoroid experiments: Interplanetary and near-Saturn. J. Geophys. Res. 85, 5841-\$\$52.

JACKSON. A. A.. AND H. A. Zook 1989. A Solar System dust ring with the Earth as its shepherd. Nature 337, 629-631.

JACKSON, A. A.. AND H. A. Zook 1992. Orbital evolution of dust particles from comets and asteroids. Icarus 97, 70-84.

JewitT. D., and J. LuU 1992. $1992 Q B_{1}$. IAU Circular No. 5611.

JtwIT, D., AND J. LL: 1993. Discovery of the candidate Kuiper belt object $1992 \mathrm{QB}_{1}$. Valure 362. 730-732.

JEWITT. D., AND J. LLC 1995. The Solar System beyond Neptune. Astron. f. 109. 1867-1876.

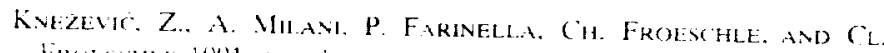

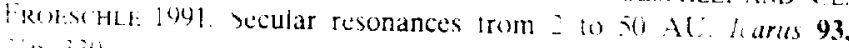
$\because n-i n$

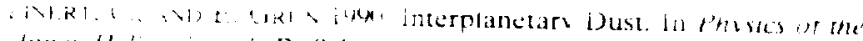
therer Helosphere / R. Schwenn and E. Marsch. Eds.). pp. 207-275. Springer-Verlag. New York.

Levasseur-Regolrd. A. C., J. B. Renard, ayd R. Dimoni 1991. The zodiacal cloud complex. In Origin and Evolution of Interplanetary Dust (A. C. Levasseur-Regourd and H. Hasegawa, Eds.). pp. 131-138. Kluwer, Dordrecht.

Levison. H. F., AND M. DUNCAN 1993. The gravitational sculpting of the Kuiper belt. Astrophys. J. 406. L35-L38.

Liou. J. C. 1993. Dynamical Evolution of Asteroidal and Cometary Particles and Their Contribution to the Zodiacal Cloud. Ph.D. dissertation, University of Florida.

Liou. J. C., S. F. DeRmott. AND Y. L. XU 1995a. The contribution of cometary dust to the zodiacal cloud. Planet. Space Sci. 43, 717-722.
Liov. J. C.. AND H. A. ZOOK 1995. An asteroidal dust ring of micronsized particles trapped in the $1: 1$ mean motion resonance with Jupiter Icarus 113, 403-414

Liov. J. C.. H. A. Zorok. AND A. A. JACKson 1995b. Radiation pressure. Poynting-Robertson drag. and solar wind drag in the $t$. Icted th $L^{2}$ body problem. Larus 116, 186-201.

Mariani. F.. and F. M. Neubauer 1990. The interpl. . ary magri:uc field. In Physics of the Inner Heliosphere I(R. Schwen: ..d E. Marsch. Eds. .. pp. 183-206. Springer-Verlag, New York.

ÖPIK. E. J. 1963. The stray bodies in the Solar System: Part 1. Survival of cometary nuclei and the asteroids. Adv. Astron. Astrophys. 2. 219-262.

Quinn. T.. S. Tremaine, anis M. Duncan 1990. Planc ... perturbations and the origin of short-p id comets. Astrophys. I :5, 667-679.

Reach, W. T.. B. A. Frav., J. L. Weiland, M. G. Hauser. T. N. Kelsall, E. L. Wright, G. Rawley, S. W. Stemwedel. and W. J. SPIESMAN 1995. Observational confirmation of a circumsolar dust ring by the COBE satellite. Vature 374, 521-523.

SANDFORD. S. A. 1986. Solar flare tract densities in interplanetarv dust particles: The determination of asteroidal versus cometary source of the zodiacal dust cloud. Karus 68. 377-34.4.

ScHWFis. R. 1990. Large-scale structure of the interplanetary medium In Pheses of the inner Hediosphere / $\mathrm{R}$. Schwenn and $\mathrm{f}$ Varsen.

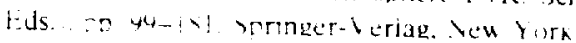

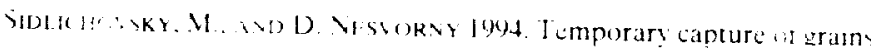
in exterior resonances with the Earth: Planar circular restricted three body rroblem with Poynting-Robertson drag. Astron. Astrophys 289, 972-982.

STERN. S. A. 1995. Collisional time scales in the Kuiper disk and their implications. Astron. J. 110, 856-868.

STERN. S. A. $1 \%$ h. Signatures of collisions in the Kuiper d. ... Astron. Astrophys., in press.

WHIPPLE. F. L. 1967. On maintaining the meteoritic complex. In The Zodiacal Light and the Interplanetary Medium (J. L. Weinberg. Ed.). pp. 409-426. NASA SP-150.

Wratt. S P., AND F. i. Whipple 1950. The Poynting-Robertson effect on meteor orbits. Astrophys. J. 111, 134-141. 